\title{
Seroprevalence and Associated Risk Factors of Toxoplasmosis among Females of Qassim University, Qassim, KSA
}

\author{
Suzan F. Ali ${ }^{{ }^{*}}$, Nagwa El Amin ${ }^{1}$, Ghaida B. AlQefari ${ }^{2}$, Ghayda S. Alwahbi ${ }^{2}$, \\ Amal I. Aljaithen ${ }^{2}$, Feda S. Alsuhibani ${ }^{2}$, Daliyah F. Alotaibi ${ }^{2}$ and Shahad S. Aljohani ${ }^{2}$ \\ ${ }^{1}$ Department of Pathology and Microbiology, College of Medicine, \\ University of Qassim, Buraidah, Saudi Arabia \\ ${ }^{2}$ Department of Pathology, College of Medicine, \\ University of Qassim, Buraidah, Saudi Arabia \\ *Corresponding author
}

\section{Keywords}

Seroprevalence,

Toxoplasma gondii,

Risk Factors,

Qassim, Saudi

Arabia

Article Info

Accepted:

08 January 2020

Available Online:

10 February 2020

\section{A B S T R A C T}

Toxoplasmosis has worldwide distribution; however, its prevalence varies within countries and communities in the same region. No studies were conducted on the seroprevalence of Toxoplasma gondii infection in Qassim region in Saudi Arabia. Our study aimed to determine the seroprevalence of toxoplasmosis among females in Qassim University, Al-melida campus, Qassim and identify the risk factors associated with Toxoplasmosis. A cross-sectional study, recruiting a random sample of 304 healthy females at Al-melida campus, Qassim was conducted on September 2018. A questionnaire was used to collect sociodemographic data of the participants and establish the risk factors leading to Toxoplasma infection. Blood samples were collected from the participants to detect $T$. gondii antibodies namely immunoglobulin $\mathrm{G}$ ( $\mathrm{IgG}$ ) using the enzyme-linked immunosorbent assay technique. A descriptive analysis of data was done to correlate between seroprevalence and risk factors. Out of 304 females 247 (81.2\%) were negative to T. gondii IgG, while 57 (18.8\%) were positive. Analysis of risk factors showed statistically insignificant association with Toxoplasma seroprevalence. The seroprevalence of $T$. gondii among females in our study is $18.8 \%$. No association between risk factors and seropositivity. For future studies, research on a large sample size is required for more accurate seroprevalence.

\section{Introduction}

T. gondii is a zoonotic sporozoan parasite, infecting warm blooded animals including humans, the definitive host is the cat (Dubey,
2010). Infection may be congenital or acquired, which can result from the consumption or handling of undercooked or raw meat containing tissue cysts, drinking unpasteurized milk or untreated water, lack of 
hand hygiene after direct contact with garden soil (Murray et al., 2013) and blood transfusion. Alternatively, it can result from direct contact with cats or from the ingestion of food contaminated by oocysts excreted in the feces of infected cats (Dubey, 2010; Murray et al., 2013). People at high risk of getting $T$. gondii infection are those who have pets especially cats, and those who live in rural areas.

Prior studies investigated high-risk groups such as pregnant women. Primary infection during pregnancy can cause severe damage to fetus and newborns including stillbirth, abortion and blindness. Immunocompetent individuals infected with $T$. gondii are generally asymptomatic, but in individuals with compromised immune systems such as patients with AIDS, chemotherapy or organ transplantation severe infections may lead to death (Torgerson et al., 2013).

Toxoplasmosis diagnosis may be achieved by serologic tests, polymerase chain reaction and histological examination of infected tissues or by isolation of the organism. Serological diagnosis of $T$. gondii infection relies on testing serum for $T$. gondii $\mathrm{IgG}$ and $\mathrm{IgM}$ antibodies because Toxoplasmosis manifests no clinical signs in $80 \%$ of cases in immunocompetent patients (Florence RobertGangneux et al., 2012; Fuccillo, 1987). Initial evaluation in the immunocompetent patients involves screening for IgG antibodies that indicates previous infection and $\operatorname{IgM}$ antibodies for the recent one, which is best measured by enzyme- linked immunosorbent assay (ELISA) method (Montoya and Liesenfeld, 2004). Polymerase chain reaction (PCR) has been developed to detect Toxoplasma DNA in clinical samples (Bastien, 2002).

It has been reported that approximately one third of the world's population is infected with T. gondii (Torgerson et al., 2013). However, this high prevalence varies from country to country (from $0-80 \%$ ), even across different communities within the same area. In a Pakistani study of pregnant women seroprevalence of $T$. gondii was found to be 19.4\% (Nazir et al., 2017). In Egypt during 2013, 2014 a case control study showed that the seroprevalence of toxoplasmosis in highrisk pregnancy group was $50.8 \%$ and the low risk group was 5\% (Kamal et al., 2015), while in Lebanon, a retrospective cohort study showed a much higher prevalence of $82.6 \%$ (Nahouli et al., 2017). Studies in Saudi Arabia revealed a prevalence of $28.5 \%$ in Eastern region (Elsafi et al., 2015), 35\% in Makkah (Ghazi et al., 2002), 38.8\% in Southwestern region (Almushait et al., 2014) and 38\% in Riyadh (Almogren et al., 2011). The most recent study was done in Tabuk University in 2017 which showed that $9.4 \%$ were positive for $T$. gondii infection (Alzaheb and Al-Amer, 2017).

Despite the published results showing high prevalence of Toxoplasma infections in regions of the kingdom of Saudi Arabia that are socioeconomically similar to Qassim region, no studies have been done in Qassim region. Therefore, there is great demand to determine the seroprevalence of toxoplasmosis among females in Qassim region.

\section{Materials and Methods}

A cross-sectional study was carried out in Melida campus at the University of Qassim, Saudi Arabia in 2018-2019. The study population involved apparently healthy female students and employees in the campus.

A random sample has been selected based on standard calculations to represent the population of Melida campus at the University of Qassim, Saudi Arabia. The 
representative sample of the population was calculated as shown below:

$$
\mathrm{N}=\mathrm{Z}^{2} \mathrm{pq} / \mathrm{d}^{2}
$$

Total population of Al-melida campus is 3339.

\section{Survey questionnaire}

Data had been collected directly from the participants by filling a validated questionnaire (Alzaheb and Al-Amer, 2017) which includes questions about sociodemographic data that involves age, marital status, monthly income and residence. We have also collected information to assess the relation between risk factors and Toxoplasma infection rate such as eating uncooked meat or contaminated fruit and vegetables, drinking unpasteurized milk or water contaminated with cat feces and exposure to garden soil without proper hygiene. A minor modification has been made on the questionnaire [a question about Grade point average (GPA) was deleted].

\section{Blood sample collection and analysis}

Venous blood samples were collected from the participants by well-trained nurses, after they have signed a consent form. Blood samples were immediately transferred to the college research laboratory. At the laboratory sera were separated from blood samples and kept at $-20^{\circ} \mathrm{C}$ till further analysis performed. Sera were tested by an indirect ELISA for detection of $T$. gondii antibodies using SERION ELISA classic T. Gondi IgG kit (Institut Viriin/ Serion GmbH; Wurzburg).

The test strips of the SERION ELISA classic microtiter plate are coated with specific antigens of the $T$. gondii. Duplicate of standard sera were included in each run and their optic densities are measured and used in calculating the samples optical densities. SERION ELISA classic T. gondii IgG was shown to have a sensitivity of $98.2 \%$ and specificity of $99.4 \%$ by the manufacturing company Elisa-antibody.com (2018). For the statistical analysis, the IBM. SPSS version 21.0 was used. Descriptive statistics have been employed to summarize the data. ChiSquare test has been used to determine if there is a significant relationship between two nominal (categorical) variables. Al-Qassim regional review board approval has been obtained for this study before carrying on with it. Participants have signed an informed consent that explained the purpose of the study and the rights of the participant for confidentiality and to withdraw at any time without any obligation towards the study team. Participant's anonymity has been assured by assigning each participant with a code number for the purpose of analysis only.

\section{Results and Discussion}

A total of 304 female participants attending or working at university of Qassim aged between 17 and 53-year-old (mean age = 21.95 years, SD: 5.025) were enrolled in this study. Most of participants reside in Buraidah (Figure 1) and their parents attained different levels of education as shown in figure 2. More than $75 \%$ of the participants have household income of $10000 \mathrm{SAR}$ or more as detailed in Figure 3.

Out of 304 females tested, 245 (81.2\%) were negative to anti $T$. gondii specific IgG antibody, (Negative results with SERION ELISA classic Toxoplasma IgG have a concentration of less than $10 \mathrm{IU} / \mu \mathrm{l})$. While $57(18.8 \%)$ were found to have anti $T$. gondii specific IgG antibody which indicates infection (figure 4). Concentrations of $T$. gondii IgG for the positive samples were calculated from the standard curve and ranged from 10$490 \mathrm{IU} / \mu \mathrm{l}$ (table 1). As illustrated in table 2 there is no clear association between studied risk factors and seropositivity ( $\mathrm{P}$ values $>0.05$ ). 
Table.1 Optical densities (ODs) and concentrations of of T. gondii IgG of Positive samples

\begin{tabular}{|c|c|c|}
\hline Sample \# & OD & $\begin{array}{c}\text { Concentration } \\
\text { IU } / \mu \mathrm{l}\end{array}$ \\
\hline 2 & 0.20 & 12.5 \\
\hline 3 & 0.19 & 12 \\
\hline 5 & 0.40 & 32 \\
\hline 7 & 0.38 & 27 \\
\hline 9 & 0.21 & 12.5 \\
\hline 10 & 0.20 & 11 \\
\hline 14 & 0.20 & 12.5 \\
\hline 18 & 0.21 & 12.5 \\
\hline 22 & 0.26 & 20 \\
\hline 23 & 1.27 & 452 \\
\hline 25 & 1.31 & 453 \\
\hline 26 & 0.27 & 20 \\
\hline 29 & 1.04 & 150 \\
\hline 30 & 0.22 & 14 \\
\hline 42 & 0.18 & 10 \\
\hline 43 & 0.21 & 14 \\
\hline 51 & 0.19 & 11 \\
\hline 52 & 0.18 & 10 \\
\hline 53 & 0.24 & 15 \\
\hline 55 & 0.26 & 20 \\
\hline 58 & 0.32 & 25 \\
\hline 59 & 0.19 & 11 \\
\hline 60 & 0.25 & 19 \\
\hline 64 & 0.25 & 19 \\
\hline 65 & 0.99 & 148 \\
\hline 74 & 0.36 & 28 \\
\hline 77 & 0.98 & 148 \\
\hline 90 & 0.22 & 14 \\
\hline 98 & 0.24 & 19 \\
\hline 99 & 0.47 & 36 \\
\hline 100 & 0.43 & 34 \\
\hline 101 & 0.17 & 10 \\
\hline 107 & 1.32 & 480 \\
\hline 113 & 1.00 & 150 \\
\hline 135 & 0.97 & 147 \\
\hline 136 & 0.83 & 96 \\
\hline
\end{tabular}


Int.J.Curr.Microbiol.App.Sci (2020) 9(2): 1482-1491

\begin{tabular}{|c|c|c|}
\hline 142 & 0.22 & 14 \\
\hline 145 & 0.26 & 20 \\
\hline 155 & 0.61 & 55 \\
\hline 170 & 1.04 & 150 \\
\hline 174 & 0.18 & 13 \\
\hline 184 & 0.68 & 65 \\
\hline 204 & 0.21 & 14 \\
\hline 207 & 0.19 & 14 \\
\hline 216 & 1.18 & 280 \\
\hline 222 & 0.89 & 110 \\
\hline 225 & 0.99 & 148 \\
\hline 227 & 0.20 & 14 \\
\hline 249 & 0.17 & 13 \\
\hline 250 & 0.18 & 13 \\
\hline 253 & 0.96 & 147 \\
\hline 255 & 0.30 & 24 \\
\hline 261 & 0.17 & 10 \\
\hline 263 & 0.27 & 20 \\
\hline 268 & 0.99 & 148 \\
\hline 277 & 1.38 & 490 \\
\hline
\end{tabular}

$60 \%$

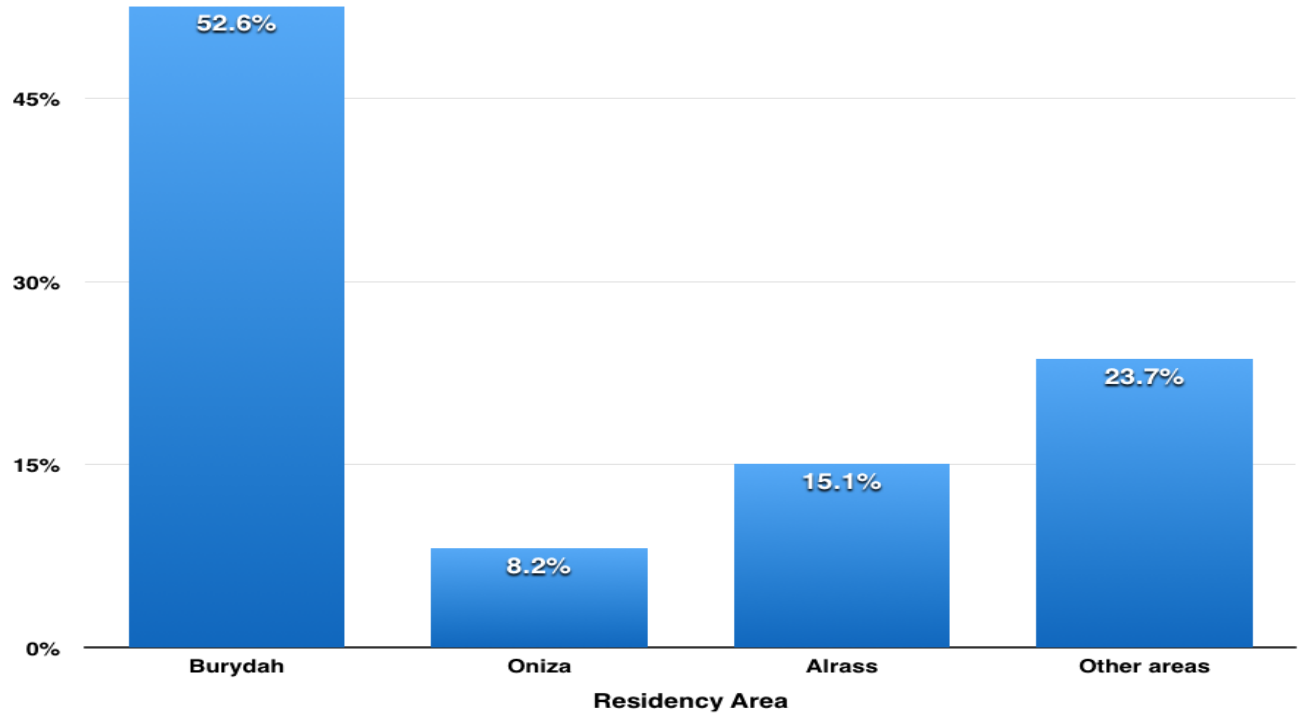

Figure .1 Most of participants reside in cities 
wather

mother

$60 \%$

$45 \%$

$15 \%$

$50 \%$

$37.5 \%$

$25 \%$

high school or less.

Figure.2 Participants parent's Education

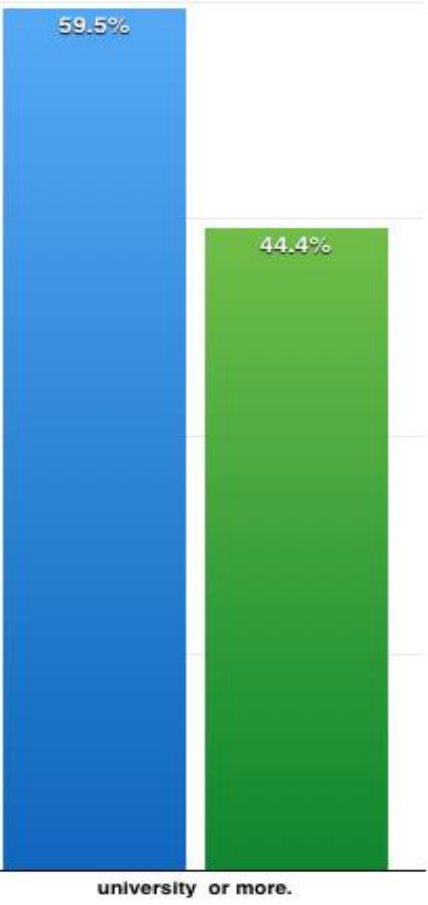

university or more.

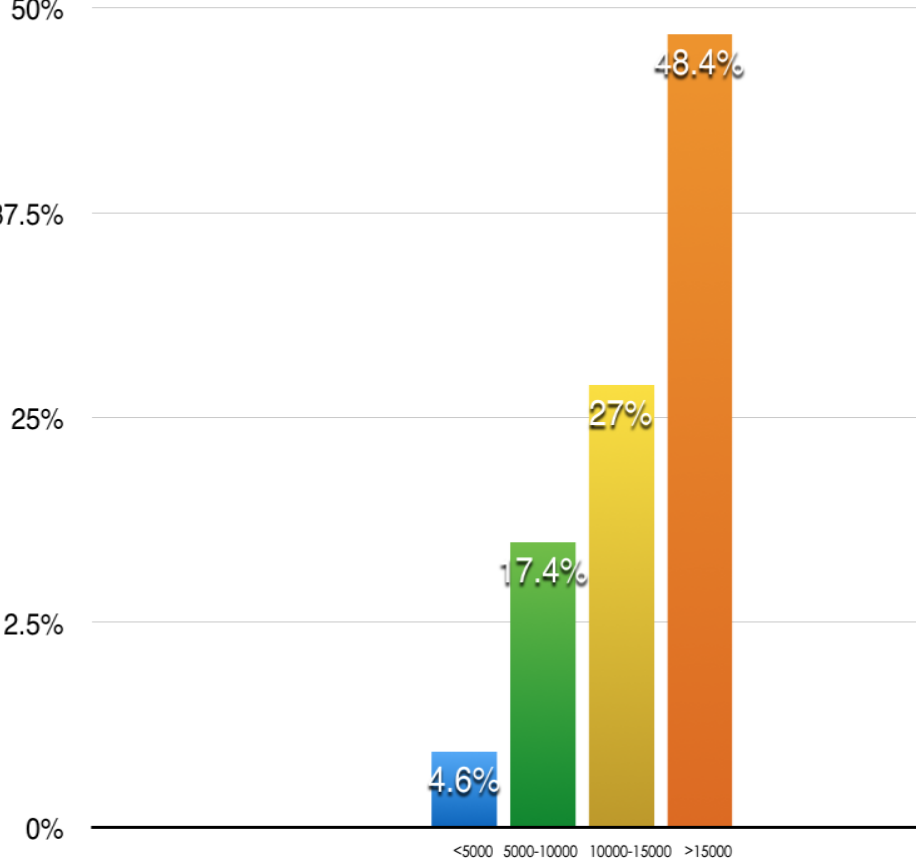

Figure.3 Monthly Income of participants 
Table.2 Analysis of the risk factors associated with seroprevalence of toxoplasmosis

\begin{tabular}{|c|c|c|c|}
\hline IgG & Positive & Negative & P value \\
\hline Residence in rural areas & 20 & 52 & 0.131 \\
\hline Monthly income less than 10.000 SAR & 12 & 55 & 0.720 \\
\hline Ownership of a cat & 12 & 56 & 0.791 \\
\hline Close contact with cats & 14 & 62 & 0.956 \\
\hline $\begin{array}{c}\text { Consumption of raw or under cooked } \\
\text { meat }\end{array}$ & 5 & 27 & 0.632 \\
\hline $\begin{array}{c}\text { Consumption of unwashed fruit \& } \\
\text { vegetables }\end{array}$ & 3 & 21 & 0.410 \\
\hline Consumption of unpasteurized milk & 4 & 18 & 0.882 \\
\hline $\begin{array}{c}\text { Do not wash after contact with raw } \\
\text { meat }\end{array}$ & 4 & 14 & 0.673 \\
\hline Contact with garden soil & 23 & 92 & 0.663 \\
\hline Drinking untreated water & 3 & 25 & 0253 \\
\hline
\end{tabular}

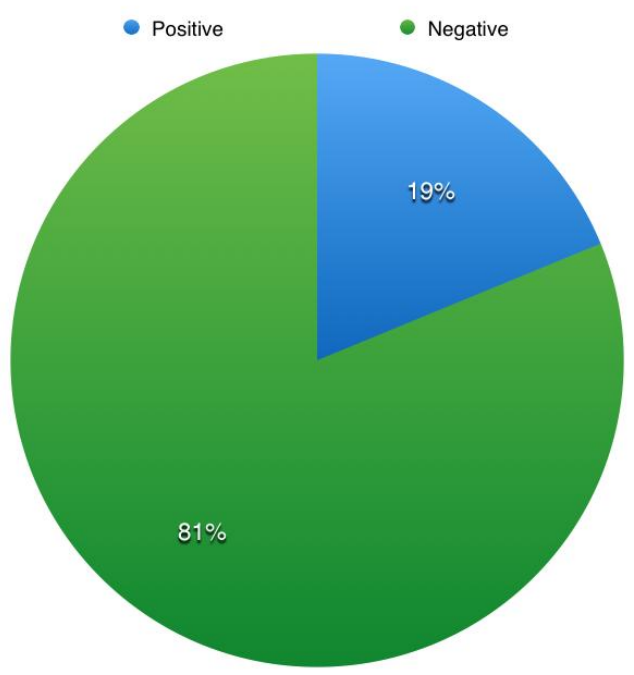

Figure.4 Seroprevalence of $T$. gondii in females

This is the first report of seroprevalence of toxoplasmosis in Qassim Region, Saudi Arabia. The aim was to determine the seropositivity of $T$. gondii infection and to assess the associated risk factors among females in Almalida campus.

The seroprevalence of $T$. gondii infection among the participants in our study is $18.8 \%$, which is less than toxoplasmosis rate of infection in most regions in Saudi Arabia, where it was found to be $35 \%$ in Makkah (Ghazi et al., 2002), 38.8\% in Southwestern region (Almushait et al., 2014) and 38\% in Riyadh (Almogren et al., 2011). 
However, the prevalence in the current study is higher than that reported from Tabuk which was only 9.4\% (Alzaheb and Al-Amer, 2017).

Generally, studies conducted in review of Toxoplasma infection rate in Saudi Arabia revealed a mean prevalence of around $30 \%$. The difference between this study and prior studies could be due to regional variation such as the climate since dry climate has less infection rates (Dubey, 2010). The difference may be attributed to the fact that our sample does not represent all categories of the region's population as the majority of participants were students. In addition, some risk factors such as eating in restaurants was not included in the questionnaire.

Although some risk factors were insignificant in this study, it has been reported that they were significant in other studies, such as living in rural areas and contact with garden soil. A study in Egypt showed increased risk of infection in rural residents, low socioeconomic status, and consumption of undercooked meat (P-value < 0.05) (Kamal et al., 2015).

The lack of association with cat contact may be because a small number of our participants are in close contact with cats and those who owned cats are well educated about cats' health and risks of contracting infection from cats.

Unexpectedly, in our study there was no significant difference in the seroprevalence of $T$. gondii between participants with high household income and those with low income this may be because of high awareness of hygienic practices among the participating group.

Unpasteurized milk also plays a role in transmission of Toxoplasma; it was not significant in this study, as most of the study population consume pasteurized milk purchased from the grocery stores.

One of the possible routes for transmission of $T$. gondii is handling or consumption of raw meat, some people may get infected through an open wound with the tissue cyst during frequent handling of raw meat of an infected animal especially if they do not wear gloves. Also, ingestion of tissue cyst is possible either from improper washing of the hands or from using contaminated cooking utensils. There is a variation in the significance of this factor among many studies, some suggest a strong relation between the consumption of undercooked meat and $T$. gondii infection such as studies in Brazil, while others found it insignificant in China (P-value $=0.496)$ and Dhahran (P-value $=0.149)$ (Elsafi et al. (2015);( Fernandes et al., (2009); Yang et al., (2017)).

Other risk factors such as consumption of unwashed fruit and vegetables and not washing hands after contact with raw meat were insignificant. These factors may have a limited role to play in the region because of the religious and cultural influences that shape dietary habits. However, being insignificant in the current study does not exclude the probability of these factors to affect toxoplasmosis transmission.

In this study we faced some limitations that could be avoided in the future. First, the sample size was calculated as 322 based on the prevalence from previous studies which was around $30 \%$. However, due to time limitation we have included 304, mainly students. Second, ELISA test has been done for anti-T. gondii specific IgG antibody that detects infection either previous or current hence further testing such as $\operatorname{IgM}$ or polymerase chain reaction will be useful in those who are at risk for complications. Third, the risk factors were evaluated using the study 
participants' questionnaire responses, which may have been affected by recall bias to some degree. Finally, this study did not assess all possible risk factors associated with $T$. gondii such as eating in restaurants.

In conclusion the prevalence of Toxoplasma infection detected in our study was relatively low compared to most parts of Saudi Arabia and higher than Tabouk. However, the prevalence is significant and further study including pregnant women is necessary considering the risk to the unborn fetuses. Moreover, the results draw attention to the need for raising awareness of toxoplasmosis, specifically with regard to the way infections occur so that women can take steps to protect themselves.

For future studies, research on a large sample size is required for more accurate seroprevalence.

\section{References}

Almogren A. 2011. Antenatal screening for Toxoplasma gondii infection at a tertiary care hospital in Riyadh, Saudi Arabia. Ann Saudi Med [Internet]. 31(6):569.

Almushait MA, Dajem SM Bin, Elsherbiny NM, Eskandar MA, Al Azraqi TA and Makhlouf LM. 2014. Seroprevalence and risk factors of Toxoplasma gondii infection among pregnant women in south western, Saudi Arabia. J Parasit Dis. 38(1):4-10.

Alzaheb RA and Al-Amer O. 2017. The Seroprevalence and Risk Factors of Toxoplasmosis among Female Undergraduate University Students in Saudi Arabia. Oman Med J [Internet]. 32(6):486-91. Available from: http://omjournal.org/articleDetails.aspx? coType $=1 \&$ aId $=2068$

Bastien P. 2002. Molecular diagnosis of toxoplasmosis. Trans. R. Soc. Trop. Med. Hyg. 96 (Suppl 1):S205-S215

Dubey JP. 2010..Toxoplasmosis of animals and humans. 2. Boca Raton: CRC Press;

Elisa-antibody.com. Indirect ELISA [Internet] [Internet]. 2018 [cited 2018 Apr 4]. Available from: http://www.elisaantibody.com/ELISA-

Introduction/ELISA-types/indirect-elisa

Elsafi SH, Al-Mutairi WF, Al-Jubran KM, Abu Hassan MM and Al Zahrani EM. 2015. Toxoplasmosis seroprevalence in relation to knowledge and practice among pregnant women in Dhahran, Saudi Arabia. Pathog Glob Health [Internet]. 109(8):377-82. Available from:

http://dx.doi.org/10.1080/20477724.201 5.1103502

Fernandes GCVR, Azevedo RS, Amaku M, $\mathrm{Yu}$ ALF and Massad E. 2009. Seroepidemiology of Toxoplasma infection in a metropolitan region of Brazil. Epidemiol Infect. 137: 180915. doi: 10.1017/S0950268809002799.

Florence Robert-Gangneux, and Marie-Laure Dardé. Epidemiology of and Diagnostic Strategies for Toxoplasmosis. 2012. Clin Microbiol Rev. 25(2): 264-296.

Fuccillo DA, Madden DL, Tzan N and Sever JL. 1987. Difficulties associated with serological diagnosis of Toxoplasma gondii infections. Diagn Clin Immunol. $5(1): 8-13$.

Ghazi HO, Telmesani AM and Mahomed MF. 2002. TORCH Agents in Pregnant Saudi Women. Med Princ Pract [Internet]. 11(4):180-2. Available from: https://www.karger.com/Article/FullTex t/65813

Kamal AM, Ahmed AK, Abdellatif MZM, Tawfik $M$ and Hassan EE. 2015. Seropositivity of toxoplasmosis in pregnant women by ELISA at Minia university hospital, Egypt. Korean J Parasitol. 53(5):605-10. 
Montoya JG, Liesenfeld O. 2004. Toxoplasmosis. Lancet 363:1965 $-1976$

Murray PR, Rosenthal KS and Pfaller MA. Medical Microbiology. 2013. In: 7th ed. Philadelphia: ELSEVIER; p. 767-8.

Nahouli H, El Arnaout N, Chalhoub E, Anastadiadis E and El Hajj H. 2017. Seroprevalence of Anti- Toxoplasma gondii Antibodies among Lebanese Pregnant Women. Vector-Borne Zoonotic Dis [Internet]. 17(12):785-90. Available from: http://online.liebertpub.com/doi/10.108 9/vbz.2016.2092

Nazir MM, Akhtar M, Maqbool A, Waheed A, Sajid MA, Ali MA, et al., 2017. Antibody Prevalence and Risk Factors for Toxoplasma gondii Infection in Women from Multan, Pakistan.
Zoonoses Public Health [Internet]. 64(7):537-42. Available from: http://www.ncbi.nlm.nih.gov/pubmed/2 8067995\%0Ahttp://doi.wiley.com/10.11 11/zph.12336

Torgerson PR and Mastroiacovo P. 2013. The global burden of congenital toxoplasmosis: a systematic review. Bull World Health Organ [Internet]. 91(7):501-8. Available from: http://www.who.int/entity/bulletin/volu mes/91/7/12-111732.pdf

Yang N, Wang D, Xing M, Li C, Li J, Wu A, et al., 2017. Seroepidemiology and risk factors of Toxoplasma gondii infection among the newly enrolled undergraduates and postgraduate students in China. Front Microbiol. $8(\mathrm{OCT})$.

\section{How to cite this article:}

Suzan F. Ali, Nagwa El Amin, Ghaida B. AlQefari, Ghayda S. Alwahbi, Amal I. Aljaithen, Feda S. Alsuhibani, Daliyah F. Alotaibi and Shahad S. Aljohani. 2020. Seroprevalence and Associated Risk Factors of Toxoplasmosis among Females of Qassim University, Qassim, KSA. Int.J.Curr.Microbiol.App.Sci. 9(02): 1482-1491. doi: https://doi.org/10.20546/ijcmas.2020.902.171 